\title{
Perfil del psicópata institucionalizado sin comportamiento criminal previo a su entrada en prisión
}

\section{A Profile of Institutionalized Psycopaths without a History of Criminal Behavior before Incarceration}

\author{
Beatriz Pérez \\ Núcleo de Ciencias Sociales y Humanidades, Universidad de La Frontera, Chile \\ Francisco Javier Rodríguez-Díaz \\ Juan Herrero \\ Asunción Fernández-Suárez \\ Departamento de Psicología, Universidad de Oviedo, España
}

Rec (03 julio 2014) Acept (29 enero 2016)

\begin{abstract}
Resumen
La inclusión en el PCL-R del comportamiento criminal previo como criterio para la evaluación de la psicopatía en las prisiones, supone que los individuos evaluados como psicópatas en la comunidad por sus consistencias de personalidad no sean detectados al llegar a prisión. Se pretende establecer el perfil diferencial del psicópata no detectado frente al psicópata criminal a nivel familiar, educativo, de consumo y delictivo. La muestra está conformada por 352 internos en prisión que fueron evaluados mediante el PCL-R. Los resultados obtenidos confirman que el psicópata no detectado presenta mismas consistencias de personalidad que el psicópata criminal, difiriendo en el estilo de vida; exhibe variables moderadoras durante su desarrollo que le han mantenido alejado del comportamiento criminal; no ha tenido un estilo de vida delictivo, pero mantiene la misma capacidad que el psicópata criminal para la comisión de los delitos más graves, siendo víctimas más frecuentes familiares y parejas.

Palabras clave: Psicopatía, conducta criminal, perfil, evaluación, variables moderadoras.
\end{abstract}

\begin{abstract}
The previous criminal behavior included in the PCL-R like a criterion to evaluation of the psychopathy in the prisons, suppose that individuals considered as psychopaths in the community for their personality were not detected when they arrive to prison. The aim of this research was to establish the different profile of the undetected psychopath in comparison with criminal psychopath in the familiar, educative, substance abuse and criminal environments. The sample was made up of 352 prisoners evaluated with the PCL-R. Results revealed that undetected psychopath showed the same personality but different lifestyle than criminal psychopath. Moreover, undetected psychopath accounted with moderating variables during his development that avoid them committing criminal behavior. Finally they did not show a criminal lifestyle, however, as the same as a criminal psychopath, they had the capacity to commit serious crimes being their victims their relatives or couples.

Keywords: Psychopathy, criminal behaviour, profile, evaluation, moderating variables.
\end{abstract}

\footnotetext{
Correspondencia: beatriz.perez@ufrontera.cl,Francisco Salazar 1145, Temuco, IX Región, gallego@uniovi.es, olaizola@uniovi.es, fernandezasuncion@
} uniovi.es, Plaza Feijoo, 33003 Oviedo, Asturias. 


\section{Introducción}

La psicopatía, como constructo caracterizado por un profundo déficit afectivo y falta de respeto por los derechos del otro (García et al., 2008; Hare, 2003; Torrubia, Poy, Moltó, Grayston y Corral, 2010), se ha convertido en uno de los trastornos de personalidad que más atención ha recibido. Debido a su histórica relación con el comportamiento criminal no es de extrañar que la construcción del concepto y el perfil del psicópata, así como la investigación sobre este cuadro clínico, se encuentre centrada desde sus inicios en el psicópata encarcelado. Frente a ello, se tiene la certeza de la existencia del trastorno fuera de la prisión, estimada su prevalencia en la población entre un 1\% y un 3\% (Hare, 1993; Torrubia y Cuquerella, 2008; Torrubia et al., 2010).

Esta línea no prioritaria es aquella que el psiquiatra Philippe Pinel (1801), autor al que se le atribuye el origen del término de psicopatía, tomó como base desde la clientela que llegaba a su consulta privada. Lo mismo ha sucedido en el caso del psiquiatra norteamericano Hervey Cleckley (1976) -ejercía su profesión en un hospital psiquiátrico para veteranos de guerra-, quien ha aportado la primera definición comprensible sobre este cuadro clínico; asume una conducta antisocial menor cometida por sus pacientes, que solían proceder de un estatus socioeconómico alto y disfrutaban de apoyo familiar y social, siendo esta conducta antisocial de naturaleza no violenta y aparentemente irracional, como subyacente a un trastorno mental (Patrick, Fowles y Krueger, 2009). Así, Cleckley advirtió de la existencia de una manifestación subclínica de este trastorno - psicópata subclínico-, refiriéndose a sujetos que presentaban las características emocionales e interpersonales del psicópata, pero que sin embargo no presentaban conducta criminal; se podían localizar adaptados, ocupando posiciones sociales respetadas, considerándolos como más competente como psicópata; es decir, a pesar de su afasia semántica, son capaces de fingir emociones que solo conocen a nivel intelectual, de manera más creíble y con mejores resultados que aquellos psicópatas que van a prisión (Cleckley, 1976). El psicópata no carcelario aprende a moverse por la sociedad sin entender los sentimientos (Garrido, 2004).

Más adelante, Robert D. Hare (1993), en su libro Without conscience (Sin conciencia), definió a este mismo grupo de sujetos como "psicópatas predelincuentes" o "subcriminales": la conducta de estos, aunque no ilegal, desafía los criterios éticos que tienen la mayoría de las personas. También se les ha llamado "psicópatas de éxito" (Babiak y Hare, 2006), "psicópatas integrados" (Garrido, 2004) y "psicópatas no encarcelados", "no institucionalizados" o "no criminales" (Hall y Benning, 2006).

El estudio de este tipo de psicopatía identificado presenta deficiencias tanto a nivel metodológico como conceptual. A nivel metodológico, la medida de la psicopatía fuera de las prisiones supone una dificultad añadida; por definición, los psicópatas no consideran tener un problema, no tienen voluntad para cambiar y creen ser perfectos tal como son, por lo que el psicópata en la comunidad, difícilmente acudirá al sistema sanitario solicitando atención. Esto impide conformar muestras de estudio que permitan conocer en mayor profundidad a este trastorno, siendo varias las aproximaciones a nivel conceptual que tratan de definir correctamente este constructo (López, 2010). Destacamos, por su implicación para este estudio, una: Expresión del trastorno influida por variables moderadoras. Esta aproximación considera que tanto el psicópata criminal como el no institucional presentan una misma etiología y una patología de la misma gravedad, siendo la conducta antisocial una consecuencia primaria en ambos casos. La diferencia radica en que en el caso de los psicópatas no encarcelados se presenta una serie de variables moderadoras (oportunidades educativas, talento, inteligencia, nivel económico, socialización efectiva, etc.) que influyen sobre la expresión conductual de los rasgos subyacentes (Garrido, 2004; Lykken, 1995). Hare (1993, p. 113), al referirse a este tipo de psicópatas, comenta que "son tan egocéntricos, insensibles y manipuladores como el resto de psicópatas; sin embargo, su inteligencia, su familia, sus habilidades sociales y sus circunstancias les permiten construir una fachada de normalidad y obtener lo que desean con relativa impunidad".

Debido a la dificultad para el estudio de este tipo de psicópatas y a la tendencia a centrarse en muestras penitenciarias, son abundantes los estudios que se han planteado como objetivo la búsqueda de factores de riesgo para el desarrollo de este trastorno o de su conducta delictiva, en detrimento de los factores moderadores o de protección (Auty, Farrington y Coid, 2015; García, Moral, Frías, Valdivia y Díaz, 2012; Krischer y Sevecke, 2008; Molinuevo, Pardo, González y Torrubia, 2014; Salekin y Lochman, 2008), también en muestras de psicópatas no institucionalizados (Lynam, Loeber, y Stouthamer-Loeber, 2008). A pesar de ello, podemos localizar algunos trabajos que determinan que el mayor número de factores de protección presentes durante la educación, es decir, una positiva socialización, favorece que individuos de alto riesgo por presentar las características de personalidad de la psicopatía, puedan evitar el contacto con el sistema penal y penitenciario (DeMatteo, 
Heilbrun y Marczyk, 2005; Hall y Benning, 2006). Además, la bibliografía tiende a relacionar las variables ambientales detectadas como factores de riesgo para el desarrollo de la psicopatía en muestras de reclusos, con la dimensión conductual de este trastorno y no tanto con las consistencias de personalidad (Farrington, 2006; Hare, 2003; Poythress, Skeem y Lilienfeld, 2006) y una mayor relación.

Sobre la capacidad de este psicópata para la comisión de actos violentos, los estudios han concluido que aunque no es habitual que este tipo de psicópatas utilice la violencia física para conseguir lo que desea -a pesar de presentar el mismo perfil de personalidad que el psicópata carcelario (Pozueco, Romero y Casas, 2011)- y pudiendo incluso presentar menor incidencia de conductas delictivas que otros individuos no psicópatas en la comunidad (DeMatteo, Heilbrun y Marczyk, 2006), pueden llegar a utilizarla si mediante la manipulación no alcanzan sus objetivos, siendo las consecuencias fatales para las víctimas (Garrido, 2004; Pozueco, 2010). En definitiva, presentan capacidad para cometer los delitos violentos más graves: aquellos dirigidos a realizar un daño deliberado a otra persona (Juarros-Basterretxea, Robles-Fernández, Silvana, Gutiérrez y García-Cueto, 2013). Respecto a esto, estudios como los realizados por Declercq, Willemsen, Audenaert y Verhaeghe (2012), Sreenivasan, Walker, Weinberger, Kirkish y Garrick (2008) y McCarthy, Huband, Patel, Banerjee y Duggan (2012) o lo recogido por Zúñiga, Vinet y León (2011), han relacionado la comisión de los crímenes más despiadados e inhumanos, la cantidad de violencia ejercida, los peores pronósticos psicosociales y la reincidencia, con las consistencias de personalidad comunes a ambos tipos de psicópatas -destacando el no institucional por ser más competente en el desarrollo de estas consistencias comunes-en detrimento de los aspectos conductuales.

A pesar de las dificultades derivadas de esta figura del psicópata adaptado a la sociedad, ello ha dado lugar a varios instrumentos que han tratado de detectar su presencia en población no institucional (Salvador, Arce y RodríguezDíaz, 2017), eliminando o minimizando como criterio la conducta criminal. Ejemplo de ello son el LSRP (Levenson, Kiehl y Fitzpatrick, 1995), el PPI (Lilienfeld y Andrews, 1996), el PCL: SV (Hart, Cox y Hare, 1995) o el SRP-III (Paulhus, Neumann y Hare, 2006). Resulta paradójico que estos sujetos que estando en libertad serían considerados como psicópatas por sus características de personalidad, necesiten cumplir una serie de criterios criminales para que continúen siendo considerados como tales cuando cometen un delito y entran en prisión.
El Hare's Psychopathy Checklist-Revised (PCL-R; Hare, 1991, 2003) es el instrumento de mayor influencia en la actualidad para la detección de la psicopatía en el interior de las prisiones (Salvador et al., 2015). Este se ha utilizado no solo en investigación y con fines clínicos, sino también dentro del sistema de justicia criminal para influir en la toma de decisiones en sentencias (DeMatteo et al., 2014; Lloyd, Clark y Forth, 2010), la asignación de grado en prisión, de permisos temporales o de libertad condicional (Guy, Kusaj, Packer y Douglas, 2015; Moltó, Poy y Torrubia, 2000), como instrumento de valoración de riesgo de violencia futura (Harris, Boccaccini y Murrie, 2015; Olver y Wong, 2015) y para la adecuación a programas de tratamiento (Spaans, Barendregt, Haan, Nijman y de Beurs, 2011). Este agrupa dos factores: el primero refiere aspectos afectivos e interpersonales, mientras que el segundo recoge la información referida al estilo de vida y la conducta antisocial. Los ítems sobre comportamiento criminal exigen para una evaluación positiva: versatilidad criminal, aparición temprana, incapacidad para aprender de la experiencia y estados coléricos. Este nivel de exigencia para la conducta antisocial no es alcanzado por el psicópata no institucional, que ha permanecido integrado en la sociedad, comete un delito y llega a prisión: puntuará alto en el factor I del PCL-R y bajo en el factor II, por lo que no son detectados con los baremos actuales del PCL-R (Garrido, 2002), a pesar de que, como dice Hare (1993, p. 87) "estos psicópatas adultos que hasta el momento de su detención pasaban por ciudadanos ejemplares, en realidad, no aparecieron de la nada. Eran las mismas personas antes y después de su detención. Son psicópatas ahora y eran psicópatas antes".

Deberían entonces dejar de ser psicópatas integrados para convertirse en psicópatas criminales (Garrido, 2004), pero el sistema de medida más popular, en la actualidad dentro de las prisiones, pasa a evaluarlos como no psicópatas. Esta situación, para parte de los estudiosos sobre el tema, es considerada como sintomatológica de un cuadro clínico a reflejar desviación personal, no desviación social o cultural (Andrade, 2008; Cooke, Hart, Logan y Michie, 2012; Cooke, Michie, Hart y Clark, 2004; Skeem y Cooke, 2010; Vinet, 2010). Fruto de ello es el desarrollo de la estructura factorial jerárquica de 3 factores para PCL-R a manos de Cooke y sus colegas (Cooke y Michie, 2001; Cooke, Michie y Skeem, 2007), que implica suprimir los ítems sobre conducta antisocial.

En un estudio anterior (Pérez, Herrero, Velasco y Rodríguez-Díaz, 2015), tras poner a prueba mediante el Análisis Factorial Confirmatorio diferentes modelos sobre 
la estructura factorial del PCL-R, se ha comprobado que el modelo jerárquico de 3 factores con testlets (Cooke y Michie, 2001; Cooke et al., 2007) muestra un ajuste sensiblemente superior en la muestra estudiada. Esta agrupa en un factor los ítems sobre aspectos interpersonales -encanto superficial, sensación desmesurada de autovalía, mentira patológica y estafador y manipulador-, mientras el 2 factor integra los ítems afectivos -ausencia de remordimiento y sentimiento de culpa, afecto superficial y poco profundo, insensibilidad afectiva y ausencia de empatía e incapacidad para aceptar la responsabilidad de las propias acciones- $y$ el factor 3 los ítems referidos al estilo de vida -necesidad de estimulación y tendencia al remordimiento, estilo de vida parasitario, ausencia de metas realistas a largo plazo, impulsividad e irresponsabilidad-, eliminando de la escala los ítems referidos a conducta delictiva y aquellos que no saturan en ningún factor -promiscuidad sexual y varias relaciones maritales de corta duración-. Ello va a dar paso a conformar como objetivo identificar la similitud entre las características de personalidad de ambos tipos de psicópatas, estableciendo el perfil diferencial (educación, desarrollo y conducta delictiva) del psicópata subclínico que llega a prisión, y no puede ser diagnosticado por la versión tradicional del PCL-R, es decir, del psicópata no detectado en las prisiones. Se pretende identificar, pues, cuáles son las variables moderadoras que permiten a este psicópata mantenerse alejado de las Instituciones Penitenciarias y definir su estilo delictivo, lo que conlleva fuertes implicaciones.

\section{Método}

\section{Participantes}

La muestra inicial está conformada por un total de 352 internos en prisión, que ha dado paso a seleccionar para dar respuesta a los objetivos planteados a un total de 103 internos, en base a su diagnóstico como psicópata detectado o no detectado. De ellos, 56 se encontraban en el Centro Penitenciario de Villabona (Asturias), cuando fueron evaluados, 23 en el Centro de Readaptación Social Puente Grande en Guadalajara (Jalisco, México) o en el Centro de Readaptación Social Francisco J. Múgica en Morelia (Michoacán, México) y los 24 restantes se encontraban cumpliendo condena en el Centro Penitenciario de Puente Alto, localizado en el área metropolitana de Santiago de Chile. Casi la totalidad de la muestra es de género masculino
(97\%), siendo la media de edad 36 años; el 79\% ha cometido delitos violentos y el $56 \%$ son reincidentes.

\section{Instrumentos}

Todos los internos penitenciarios participantes forman parte de la muestra de manera voluntaria y anónima, solicitándoles permiso y consentimiento con anterioridad a la aplicación de las pruebas. Posteriormente se ha contado con los expedientes penitenciarios de los internos, que nos permitieron obtener información fidedigna sobre su historial penitenciario, además de informes realizados por otros profesionales sobre su historia de vida personal y la evaluación social y psicológica. A continuación, mediante la Ficha de Historia Penitenciaria (Estrada, Rodríguez y Solano, 2012) se establecen las características contextuales básicas a considerar de los individuos, en tanto responde a las principales variables criminógenas de los internados en Centros Penitenciarios: estado civil, escolaridad y actividad laboral, adicciones, carrera delictiva y familia.

A continuación se ha utilizado una adaptación española de la Entrevista Semiestructurada del PCL-R (Torrubia et al., 2010), realizada por Pérez, Herrero, Velasco y Rodríguez-Díaz (2015). Tras poner a prueba mediante el Análisis Factorial Confirmatorio los diferentes modelos sobre la estructura factorial del PCL-R, se asume el modelo jerárquico de 3 factores con testlets (Cooke y Michie, 2001; Cooke et al., 2007), que mostró ser invariante en muestra española y latinoamericana (Pérez, 2014), con un nuevo punto de corte para el diagnóstico siguiendo las mismas indicaciones utilizadas por el autor de la prueba (Torrubia et al., 2010). Finalmente, se obtuvo una nueva escala fiable de 13 ítems, con $\alpha=.85$ y $\alpha$ 's $\geq .75$ para los tres factores, siendo fiable también para la muestra utilizada en este estudio $(\alpha=.83$ y $\alpha$ 's $\geq .71$ en los tres factores) y con validez discriminante libre de conducta antisocial, que detecta al 96.5\% de los psicópatas de Hare, y a un $8.6 \%$ más. Este porcentaje de psicópatas anteriormente no detectados cumple las características esenciales del psicópata, como son la capacidad de manipulación, la ausencia de empatía y remordimientos y una deficiente afectividad, pero sin presentar una carrera criminal anterior.

\section{Procedimiento y análisis de los datos}

Con el objetivo de construir el perfil del psicópata habitualmente no detectado en las prisiones, en base a la incidencia de diferencias en variables sobre la competencia paterna para educar, variables sobre el desarrollo del menor (escolar y de 
consumo) y variables cuantitativas y cualitativas del delito, se realizado un análisis multivariado de la varianza con el paquete estadístico SPSS 19.0 para Windows. De acuerdo con la naturaleza de las variables analizadas, a su vez, se ha utilizado como técnicas estadísticas la prueba chi-cuadrado, el Análisis Univariado de la Varianza (ANOVA) y el Análisis Multivariado de la Varianza (MANOVA),

\section{Resultados}

Los participantes es este estudio fueron 352 internos en prisión, evaluados mediante la escala de Psicopatía PCL-R (Torrubia et al., 2010) de acuerdo con la versión de 13 ítems derivada de la estructura factorial jerárquica con testlets de 3 factores (Pérez et al., 2015). De estos, 100 (28.3\%) obtuvieron un diagnóstico positivo de psicopatía; 62 también eran diagnosticados por la versión tradicional de la escala, añadiéndose 3 más que no lo eran por la versión de 3 factores. El grupo final es de 65 internos (18.5\%) de psicópatas detectados, que equivaldría al psicópata criminal de R. D. Hare. Los 38 restantes conformaron un segundo grupo $(10.8 \%)$, al que denominamos psicópatas no detectados, pues pasan desapercibidos para la versión tradicional del PCL-R. Estos equivaldrían a los psicópatas subclínicos o de éxitos que, estando integrados en la sociedad y $\sin$ presentar carrera delictiva anterior, cometen un delito y van a prisión. Finalmente se ha trabajado con una muestra de 103 internos.

Con el objeto de responder al objetivo planteado, en primer lugar, se pasa a comprobar si el patrón de puntuaciones obtenida en la estructura de 3 factores de Cooke y Michie (2001) es similar en ambos tipos de psicópatas o si por el contrario se detectan diferencias significativas. Para tal fin se ha utilizado el Análisis Multivariado de la Varianza (MANOVA) como técnica estadística y el estadístico Lambda de Wilks como prueba general de significación, poniendo los resultados de manifiesto diferencias significativas en relación a la distribución de las puntuaciones en esta escala en base al tipo de psicópata $($ Valor $=.721 / \mathrm{F}=12.74 / \mathrm{p}<.001)$.

$\mathrm{Al}$ observar con detenimiento los resultados del modelo, se constata que las diferencias significativas establecidas se deben al factor 3 sobre estilo de vida ( $M=7.29$ / Rango de 3 a 10/ DT $=1.66$ ), con un valor $\mathrm{p}<.001$ y tamaño del efecto (d) de .91. El psicópata detectado obtiene una puntuación más elevada en este factor, lo cual significa un estilo de vida más deteriorado. Este factor, concretamente, recoge los ítems necesidad de estimulación/tendencia al aburrimiento, estilo de vida parasitario, ausencia de metas realistas a largo plazo, impulsividad e irresponsabilidad. En lo que respecta a los factores 1 y 2 , que hacen referencia a consistencias de personalidad, no se observan diferencias significativas entre ambos tipos de psicópatas.

Atendiendo a este factor sobre estilo de vida que determina las diferencias significativas entre ambos tipos de psicópatas, sometimos a estudio una serie de variables referentes al desarrollo de carácter familiar, educativo, de consumo y delictivo. Se pretende con ello determinar la incidencia de estas variables sobre la distribución de las puntuaciones en este factor. Utilizando como técnica estadística el Análisis Univariado de la Varianza (ANOVA), encontramos: un ambiente familiar de consumo durante la infancia del interno $(\mathrm{p}=.001 ; \mathrm{d}=.38)$; tener un padre que ha sido detenido en alguna ocasión $(\mathrm{p}=.001 ; \mathrm{d}=.51)$; así como los hermanos $(\mathrm{p}=.019 ; \mathrm{d}=.29)$; la ausencia de al menos uno de sus padres durante la infancia por ingreso en prisión $(\mathrm{p}=.012 ; \mathrm{d}=.64)$; una negativa $\mathrm{o}$ inexistente relación con uno o ambos padres $(\mathrm{p}=.016 ; \mathrm{d}=.27)$; la falta de un estilo educativo adecuado durante la infancia y adolescencia -autorizativo- $(\mathrm{p}=.008 ; \mathrm{d}=.34)$; y una crianza, al menos en parte, a manos de instituciones, con padres de acogida o adoptivos o solos en la calle $(\mathrm{p}=.002 ; \mathrm{d}=.63)$ favorece la obtención de una puntuación más elevada en el factor 3 sobre estilo de vida. Una misma tendencia se observa con variables educativas y de consumo: Nivel de estudios bajo -estudios secundarios inacabados o inferiores- $(\mathrm{p}<.001 ; \mathrm{d}=.48)$; problemas graves con los profesores durante la escuela, siendo el interno el agresor $(\mathrm{p}<.001 ; \mathrm{d}=.53)$; o con los compañeros $(\mathrm{p}=.001 ; \mathrm{d}=.42)$; la adicción a drogas ilegales $(\mathrm{p}<.001 ; \mathrm{d}=.83)$; y el policonsumo $(\mathrm{p}<.001 ; \mathrm{d}=.31)$, se asocia con puntuaciones más elevadas en el factor 3 .

Un estilo de vida deteriorado se relaciona también con un estilo de vida delictivo. Mediante el Análisis Univariado de la Varianza (ANOVA), comprobamos que: la comisión de delito no sancionado antes de la primera detención $(\mathrm{p}=.001 ; \mathrm{d}=.51)$; y un mayor número de entradas en prisión $(\mathrm{p}<.001 ; \mathrm{d}=.46)$ favorece la obtención de peores puntuaciones en este factor. En referencia al tipo de infracción, la comisión de una gran variedad de delitos se asocia con un estilo de vida impulsivo e irresponsable en la edad adulta: delitos de conducción ( $\mathrm{p}=.002 ; \mathrm{d}=.45)$; económicos $(\mathrm{p}=.015 ; \mathrm{d}=.34)$; de robo no violentos como hurtos o robos con fuerza $(\mathrm{p}<.001 ; \mathrm{d}=.50)$; u otros no violentos como los daños a la propiedad o el vandalismo $(\mathrm{p}=<001 ; \mathrm{d}=.50)$; delitos de robos con violencia ( $\mathrm{p}$ $<.001 ; \mathrm{d}=.70)$; delitos de lesiones o atentado contra la autoridad $(\mathrm{p}<.001 ; \mathrm{d}=.65)$; de homicidio/asesinato o de intento de homicidio/asesinato $(\mathrm{p}=.009 ; \mathrm{d}=.35)$; sexuales 
contra adultos $(\mathrm{p}=.049 ; \mathrm{d}=.55) ; \mathrm{y}$ otros delitos violentos como amenazas o posesión de arma de fuego sin licencia $(\mathrm{p}<.001 ; \mathrm{d}=.60)$.

Considerando las abundantes relaciones significativas establecidas en base a las puntuaciones obtenidas en este factor 3 -siendo este factor en el que se fundamentan las diferencias entre el psicópata detectado y el no detectado-, decidimos comprobar si estas son extrapolables al diagnóstico de psicopatía. Los resultados obtenidos sobre el análisis diferencias de la variable tipo de psicópata (detectado o no detectado) en relación a las variables familiares, del desarrollo y delictivas, ponen de manifiesto diferencias significativas. En la Tabla 1 se presentan los resultados obtenidos mediante el Análisis Univariado de la Varianza (ANOVA) para las variables cuantitativas, y en la Tabla 2 los resultados obtenidos mediante el análisis chi-cuadrado para las de carácter cualitativo.

El psicópata no detectado abandona la escuela a una edad más tardía y además se inicia en el consumo de tabaco -droga de iniciación - a una media de 15,8 años, mientras que el psicópata detectado lo hace antes de alcanzar los 13 .
Su conducta antisocial se entiende en menor medida como un estilo de vida, ya que su carrera delictiva comienza a una edad más tardía: la edad del primer delito no sancionado, la de la primera entrada en prisión y la de primer ingreso, es significativamente superior a la que presenta el psicópata detectado. Además, entra en prisión un menor número de veces -el psicópata no detectado presenta una media de 1.64 entradas mientras que el detectado alcanza una media de 5- y el tiempo total de condena en prisión es más corto (Véase tabla).

El psicópata no detectado, a diferencia del psicópata detectado (criminal), ha crecido en mayor medida en una familia sin consumo abusivo de alcohol u otras drogas de carácter ilegal por parte de padres o hermanos, en la que se han establecido normas y límites transmitidos con afecto y diálogo (estilo educativo autorizativo) o sin ellos (estilo educativo autoritario), sin ausencia del padre ni detenciones de los hermanos. De igual manera se establecen diferencias significativas en base a analizar la relación entre el tipo de psicópata y variables sobre el consumo. Un menor número de psicópatas no detectados son consumidores de drogas

Tabla 1. Resultado del Análisis Univariado de la Varianza (ANOVA) del efecto diferencial del tipo de psicópata sobre variables del desarrollo y variables cuantitativas del delito

\begin{tabular}{|c|c|c|c|c|c|c|}
\hline Variables del desarrollo & Media & DT & Gl & $\mathrm{F}$ & Sig. & d \\
\hline \multicolumn{7}{|c|}{ Edad abandono escolar $(\mathrm{N}=95)$} \\
\hline Psicópata detectado & 14.13 & 3.58 & 1 & 10.05 & .002 & .65 \\
\hline Psicópata no detectado & 16.91 & 4.93 & & & & \\
\hline \multicolumn{7}{|c|}{ Edad de inicio en el consumo de tabaco $(\mathrm{N}=81)$} \\
\hline Psicópata detectado & 12.83 & 4.78 & 1 & 6.041 & .016 & .55 \\
\hline Psicópata no detectado & 15.81 & 6.07 & & & & \\
\hline \multicolumn{7}{|c|}{ Variables cuantitativas del delito } \\
\hline \multicolumn{7}{|c|}{ Edad del primer delito no sancionado $(\mathrm{N}=82)$} \\
\hline Psicópata detectado & 11.64 & 3.24 & 1 & 9.28 & .003 & .66 \\
\hline Psicópata no detectado & 16.03 & 9.42 & & & & \\
\hline \multicolumn{7}{|c|}{ Edad de primera detención $(\mathrm{N}=95)$} \\
\hline Psicópata detectado & 16.72 & 8.61 & 1 & 13.27 & $<.001$ & .73 \\
\hline Psicópata no detectado & 24.80 & 12.99 & & & & \\
\hline \multicolumn{7}{|c|}{ Edad del primer ingreso penal $(\mathrm{N}=101)$} \\
\hline Psicópata detectado & 21.90 & 8.52 & 1 & 15.05 & $<.001$ & .75 \\
\hline Psicópata no detectado & 29.91 & 12.15 & & & & \\
\hline \multicolumn{7}{|c|}{ Número de entradas en prisión $(\mathrm{N}=101)$} \\
\hline Psicópata detectado & 5 & 8.65 & 1 & 5.47 & .021 & .48 \\
\hline Psicópata no detectado & 1.64 & 1.05 & & & & \\
\hline \multicolumn{7}{|c|}{ Meses de condena $(\mathrm{N}=101)$} \\
\hline Psicópata detectado & 190.56 & 130.22 & 1 & 6.145 & .015 & .49 \\
\hline Psicópata no detectado & 127.08 & 114.73 & & & & \\
\hline
\end{tabular}


Tabla 2. Resultados del análisis chi-cuadrado. Relación tipo de psicópata y variables familiares, del desarrollo (consumo) y variables cualitativas del delito

\begin{tabular}{|c|c|c|c|c|c|}
\hline Variables familiares & Detectado & No detectado & $\chi^{2}$ & Sig. & $\mathrm{d}$ \\
\hline \multicolumn{6}{|c|}{ Ambiente de consumo familiar $(\mathrm{N}=94)$} \\
\hline No & $25(43.1 \%)$ & $23(63.9 \%)$ & \multirow{2}{*}{3.840} & \multirow{2}{*}{.050} & \multirow{2}{*}{.42} \\
\hline Sí & $33(56.9 \%)$ & $13(36.1 \%)$ & & & \\
\hline \multicolumn{6}{|c|}{ Ausencia de normas y límites $(\mathrm{N}=95)$} \\
\hline No & $21(35.6 \%)$ & $27(75 \%)$ & \multirow{2}{*}{13.889} & \multirow{2}{*}{$<.001$} & \multirow{2}{*}{.80} \\
\hline Sí & $38(64.4 \%)$ & $9(25 \%)$ & & & \\
\hline \multicolumn{6}{|l|}{ Falta del padre $(\mathrm{N}=101)$} \\
\hline No & $26(40.6 \%)$ & $26(70.3 \%)$ & \multirow{2}{*}{8.249} & \multirow{2}{*}{.004} & \multirow{2}{*}{.60} \\
\hline Sí & $38(59.4 \%)$ & $11(29.7 \%)$ & & & \\
\hline \multicolumn{6}{|c|}{ Hermanos detenidos $(\mathrm{N}=94)$} \\
\hline No & $35(60.3 \%)$ & $29(80.6 \%)$ & \multirow{2}{*}{4.176} & \multirow{2}{*}{.041} & \multirow{2}{*}{.45} \\
\hline Sí & $23(39.7 \%)$ & $7(19.4 \%)$ & & & \\
\hline \multicolumn{6}{|c|}{ Variables del desarrollo (consumo) } \\
\hline \multicolumn{6}{|c|}{ Adicción a las drogas ilegales $(\mathrm{N}=101)$} \\
\hline No & $3(4.7 \%)$ & $10(27 \%)$ & \multirow{2}{*}{8.536} & \multirow{2}{*}{.003} & \multirow{2}{*}{.69} \\
\hline Sí & $61(95.3 \%)$ & $13(73 \%)$ & & & \\
\hline Consumo de marihuana $(\mathrm{N}$ & & & & & \\
\hline No & $8(12.7 \%)$ & $13(35.1 \%)$ & & & \\
\hline Sí & $55(87.3 \%)$ & $24(64.9 \%)$ & 7.073 & .008 & .53 \\
\hline Consumo de pastillas $(\mathrm{N}=$ & & & & & \\
\hline No & $31(48.4 \%)$ & $32(86.5 \%)$ & 14.463 & $<.001$ & .77 \\
\hline Sí & $33(51.6 \%)$ & $5(13.5 \%)$ & 14.403 & $<.001$ & .11 \\
\hline Consumo de inhalantes $(\mathrm{N}$ & & & & & \\
\hline No & $49(76.3 \%)$ & $37(100 \%)$ & 10184 & 001 & 45 \\
\hline Sí & $15(23.4 \%)$ & $0(0 \%)$ & 10.184 & .001 & .45 \\
\hline Variables cualitativas del delito & & & & & \\
\hline Delitos de robo sin violen & & & & & \\
\hline No & $16(24.6 \%)$ & $19(50 \%)$ & & & \\
\hline Sí & $49(15.4 \%)$ & $19(50 \%)$ & 6.888 & .009 & .53 \\
\hline Delitos de conducción & & & & & \\
\hline No & $45(69.2 \%)$ & $33(86.8 \%)$ & 4047 & 044 & \\
\hline Sí & $20(30.8 \%)$ & $5(13.2 \%)$ & 4.047 & .044 & .41 \\
\hline Otros delitos no violent & & & & & \\
\hline No & $27(41.5 \%)$ & $31(81.6 \%)$ & 15628 & $<001$ & \\
\hline Sí & $38(58.5 \%)$ & $7(18.4 \%)$ & 15.628 & $<.001$ & .81 \\
\hline Delitos de robos con viole & & & & & \\
\hline No & $18(27.7 \%)$ & $28(73.3 \%)$ & & & \\
\hline Sí & $47(72.3 \%)$ & $10(26.3 \%)$ & 20.524 & $<.001$ & .92 \\
\hline Lesiones/atentado contra la au & & & & & \\
\hline No & $17(26.2 \%)$ & $28(73.3 \%)$ & 22021 & $<001$ & 97 \\
\hline Sí & $48(73.8 \%)$ & $10(26.3 \%)$ & 22.021 & $<.001$ & .91 \\
\hline Delitos de homicidio/asesi & & & & & \\
\hline No & $37(56.9 \%)$ & $39(78.9 \%)$ & 5116 & 024 & 46 \\
\hline Sí & $28(43.1 \%)$ & $8(21.1 \%)$ & 5.110 & .024 & .40 \\
\hline Otros delitos violentos & & & & & \\
\hline No & $27(41.5 \%)$ & $29(76.3 \%)$ & 11691 & 001 & 67 \\
\hline Sí & $38(58.5 \%)$ & $9(23.7 \%)$ & 11.091 & .001 & $.0 /$ \\
\hline Delitos sexuales contra me & & & & & \\
\hline No & $59(90.8 \%)$ & $28(73.7 \%)$ & & & \\
\hline Sí & $6(9.2 \%)$ & $10(26.3 \%)$ & 5.335 & .023 & .46 \\
\hline
\end{tabular}


en comparación con los psicópatas carcelarios y, además, consume en menor medida ciertas drogas: la marihuana, las pastillas y los inhalantes, lo que no sucede con el resto de drogas estudiadas -cocaína, heroína, éxtasis, tabaco y alcohol- (Véase tabla).

Por último, lo mismo sucede cuando analizamos la relación entre tipo de psicopatía y tipo de delitos cometidos (Véase Tabla 2), donde se puede observar que el psicópata no detectado comete en menor medida delitos relacionados con un estilo de vida antisocial, como son los delitos de conducción, hurtos, robo con fuerza, intimidación o violencia, delitos de lesiones o atentado contra la autoridad, otros delitos no violentos como los daños a la propiedad o el vandalismo, y otros violentos como son las amenazas o la posesión de armas de fuego sin licencia. En lo que respecta a otros delitos como los relacionados con la salud pública o los delitos económicos, no se encuentran diferencias significativas entre los grupos. Lo mismo sucede con otros de mayor gravedad y/o violencia, como los delitos violentos contra la pareja o delitos sexuales contra adultos; los delitos sobre agresión sexual a menores se dan con mayor frecuencia entre los psicópatas no detectados, y los homicidios y asesinatos o intentos de homicidio o asesinato entre los detectados.

La bibliografía considera que el psicópata no carcelario, el no detectado, tiene la misma capacidad para cometer actos violentos que el psicópata criminal. Deteniéndonos en aquellos delitos violentos que se caracterizan por una mayor agresividad y crueldad, es decir, aquellos que tienen como finalidad producir daño o abusar de otro individuo, encontramos resultados dispares dependiendo del delito. El homicidio/asesinato o intento de homicidio/asesinato tiene mayor presencia entre el psicópata criminal. Los delitos sexuales contra menores, por el contrario, tienen mayor presencia en el psicópata no institucionalizado. Sobre los delitos sexuales contra adultos y los delitos contra la pareja (refiriéndonos a los más graves: homicidio/asesinato o intento de homicidio/asesinato y delitos sexuales) no se encontraron diferencias significativas. Ante la confusión, retomamos el total de la muestra de 352 internos y las puntuaciones obtenidas por los participantes en las facetas de la versión tradicional del PCL-R de 20 ítems: Las faceta 1 -sobre aspectos interpersonales-, 2 -sobre aspectos afectivos- $\mathrm{y}$ 3 -sobre estilo de vida-, que se corresponden con los tres factores del modelo jerárquico de tres factores y además la cuarta faceta que reúne los ítems sobre conducta delictiva, con la intención de estudiar si este tipo de delitos más graves se asocian bien con los aspectos de personalidad, comunes para ambos tipos de psicópatas, o los conductuales, en los que difieren. Utilizando como técnica estadística el Análisis Multivariado de la Varianza (MANOVA), encontramos que el estadístico Lambda de Wilks como prueba general de significación arroja un resultado significativo (Valor $=.867 / \mathrm{F}=13.29 / \mathrm{p}<.001)$. Esto indica que aquellos que cometen este tipo de delitos presentan una distribución de las puntuaciones en las facetas del PCL-R significativamente distinta a aquellos que no los cometen.

Al observar con detenimiento los valores obtenidos para cada una de las facetas, encontramos que las diferencias significativas en la distribución de las puntuaciones a nivel global se deben a las facetas $1(\mathrm{M}=3,8 /$ Rango de 0 a $8 / \mathrm{DT}=2,388)$ y $2(\mathrm{M}=4,47 /$ Rango de 0 a $8 / \mathrm{DT}=$ 2,37); aquellos internos que han cometido estos delitos de mayor crueldad y gravedad puntúan más alto que el resto de internos en las facetas que evalúan consistencias de personalidad ( $\mathrm{p}<.001$ para ambas facetas, con un tamaño del efecto para la faceta 1 de .60 y de .78 para la faceta 2), en cambio, en lo que respecta a las facetas 3 y 4 , que se centran en aspectos conductuales, no se encuentran diferencias significativas.

En este punto de los análisis y considerando la mayor calidad de la red de apoyo y la ausencia de un estilo de vida

Tabla 3. Resultados del análisis chi-cuadrado sobre la relación tipo de psicópata y vínculo con la víctima de delitos violentos graves

\begin{tabular}{|c|c|c|c|c|c|}
\hline Delitos violentos graves contra $(n=54)$ & Detectado & No detectado & $\chi^{2}$ & Sig. & $\mathrm{d}$ \\
\hline \multicolumn{6}{|l|}{ Delitos violentos graves contra familiar o pareja } \\
\hline No & $25(71.4 \%)$ & $8(42.1 \%)$ & \multirow{2}{*}{4.456} & \multirow{2}{*}{.035} & \multirow{2}{*}{.59} \\
\hline Sí & $10(28.6 \%)$ & $13(36.1 \%)$ & & & \\
\hline \multicolumn{6}{|c|}{ Delitos violentos graves contra conocido o desconocido } \\
\hline No & $7(20 \%)$ & $9(47.4 \%)$ & \multirow{2}{*}{4.424} & \multirow{2}{*}{.035} & \multirow{2}{*}{.60} \\
\hline Sí & $28(80 \%)$ & $10(52.6 \%)$ & & & \\
\hline
\end{tabular}


delictivo en el caso del psicópata no detectado, se ha decidido estudiar la posible relación entre el tipo de psicopatía y el vínculo con las víctimas de estos delitos de mayor gravedad y violencia (Véase Tabla).

Los resultados obtenidos establecen diferencias significativas en ambas variables con un tamaño del efecto medio siendo, según los residuos corregidos que arrojan los valores $\mathrm{z}$, mayor la probabilidad de que un psicópata detectado cometa un delito contra conocidos o desconocidos y mayor la probabilidad de que un psicópata no detectado cometa un delito grave contra un familiar o pareja, con un valor $\mathrm{p}$ $<.05$ en ambos casos. El psicópata detectado, el criminal, tiende a presentar un mayor número de delitos graves en los que la víctima es un desconocido o conocido con o sin relación, mientras que el psicópata no detectado presenta en mayor medida víctimas que se encuentran dentro de su círculo familiar.

\section{Discusión}

Los resultados alcanzados demuestran que este tipo de psicópata no detectado mantiene las mismas características de personalidad que el psicópata criminal, tal y como indicaba la literatura (Garrido, 2004; Hare, 1993; Pozueco, 2010; Pozueco et al., 2011), difiriendo solo en el estilo de vida: el psicópata criminal es más irresponsable e impulsivo, presenta una mayor búsqueda de sensaciones y tendencia al aburrimiento, planes de futuro menos realistas y, además, un estilo de vida parasitario. Esta forma desordenada de vida se asocia con un mayor deterioro a nivel familiar, educativo y de consumo durante el proceso de socialización, además de una carrera delictiva de inicio más temprano, más reincidente y a la comisión de un mayor tipo de delitos.

A sabiendas de que el estilo de vida es el punto de inflexión que diferencia al psicópata detectado del no detectado, tratamos de extrapolar esta relación al diagnóstico de psicopatía. Encontramos que un diagnóstico de psicópata detectado se asocia con un estilo de vida delictivo, pues como hemos visto se inicia en la conducta delictiva a una edad más temprana, es más reincidente y además el tiempo de condena acumulado en meses es mayor, lo cual es esperable considerando que el no detectado se ha mantenido hasta el momento de su detención integrado en la sociedad. El tipo de delitos cometidos también dan cuenta de estas diferencias, puesto que los delitos asociados con un estilo de vida delictual (hurtos, robos con fuerza, intimidación y violencia, delitos de lesiones y/o atentado contra la autoridad, delitos de conducción, vandalismo, posesión de arma de fuego sin licencia, etc.) tienen mayor presencia en el psicópata criminal, distando mucho del perfil delictivo del psicópata no detectado por la versión tradicional del PCL-R. Este último presenta una conducta delictiva que puede ser compaginada en mayor medida con una apariencia de normalidad: delitos contra la salud pública, económicos, contra la pareja, delitos sexuales contra menores, etc.

La literatura sobre la comisión de delitos violentos por parte del psicópata no institucionalizado y el criminal, nos dice que ambos presentan capacidad para llevarlos a cabo (Garrido, 2004; Pozueco et al., 2010). Son varios los estudios empíricos que han relacionado los actos de mayor crueldad y violencia depredadora, las peores predicciones sobre reincidencia violenta, etc., con las consistencias de personalidad -comunes en ambos-en detrimento de aspectos conductuales -en los que difieren-(Declercq et al., 2012; McCarthy et al., 2012; Sreenivasan et al., 2008). Los resultados obtenidos corroboran dichos estudios, relacionándose la comisión de delitos violentos graves contra las personas, con puntuaciones significativamente más elevadas en los ítems sobre aspectos afectivos e interpersonales, no siendo así para los ítems sobre estilo de vida y conducta delictiva. Además, a sabiendas de que el psicópata no institucionalizado mantiene una mejor integración en la sociedad (Babiak y Hare, 2006; Hare, 2003; Garrido, 2004; Pozueco, 2010) hemos comprobado que son los familiares y parejas las víctimas más habituales del psicópata no detectado en este tipo de delitos, mientras que para el psicópata criminal lo son los conocidos y desconocidos.

Finalmente, en lo que respecta a la conducta delictiva hemos encontrado delitos sexuales contra menores como un delito característico del psicópata no detectado. Este resultado es revelador en la medida en la que la figura del pedófilo o pederasta-manipulador, carente de afectividad, culpabilidad, empatía y de responsabilidad ante los actos cometidos, ha pasado habitualmente desapercibido como psicópata en el sistema penal y penitenciario en lo que respecta a la toma de decisiones sobre condenas, permisos, asignación a programas de tratamiento, etc. Aunque esta observación es extensible al resto de psicópatas no detectados cobra especial importancia en este tipo de delito, que habitualmente se ha mantenido alejado de este constructo y de las implicaciones que de este se derivan para el tratamiento.

La pregunta que ahora debemos hacernos es: ¿qué sucede en la vida de estas personas para que unos se conviertan en psicópatas criminales y otros en psicópatas subclínicos? Ciertas variables moderadoras pueden intervenir durante el proceso de socialización del psicópata, favoreciendo que exprese su fenotipo de manera distinta. La inteligencia, 
oportunidades educativas, una socialización efectiva, etc., pueden funcionar como factores de protección para el desarrollo de un estilo de vida delictivo (Garrido, 2004; Hare, 2003; López, 2010; Lykken, 1995). A pesar de que algunos trabajos han identificado aspectos familiares y del desarrollo que diferencian al psicópata criminal del resto de la población infractora (Auty et al., 2015; Farrington, 2006; García et al., 2012; Krischer y Sevecke, 2008; Molinuevo, et al., 2014; Salekin y Lochman, 2008) o al psicópata subclínico del resto de la población no institucional (Lynam et al., 2008), la investigación no ha dado respuesta a cuáles son estas variables moderadoras que influyen en el desarrollo de un tipo u otro de psicopatía.

$\mathrm{Al}$ abordar esta temática hemos identificado un menor número de factores de riesgo para la conducta delictiva en el ámbito familiar y en el desarrollo del psicópata no detectado. Se constata que ha tenido un proceso de socialización más normalizado, caracterizado por un estilo educativo autorizativo o autoritario basada en normas, límites y supervisión parental; además, no le ha faltado la figura paterna durante su infancia y/o adolescencia, no ha crecido en un ambiente permisivo con el abuso de drogas legales o ilegales ni sus hermanos han tenido contacto con el ámbito policial. También permanece en el sistema educativo durante más tiempo, consume drogas en menor porcentaje y se inicia de manera más tardía en el consumo de la droga legal de iniciación, el tabaco. Por último, aquellos psicópatas no detectados consumidores son adictos en menor medida a drogas habitualmente relacionadas con el ámbito marginal, como son la marihuana, las pastillas y los inhalantes.

Estos resultados avivan el debate sobre el sistema de evaluación utilizado para la psicopatía en el interior de las prisiones. Tal y como señalan Garrido (2004) y otros autores (Cooke et al., 2012; Patrick et al., 2009; Pérez et al., 2015; Pozueco, 2010), en las prisiones se localizan individuos que, aunque mantienen una personalidad psicopática, pasan desapercibidos para el sistema penitenciario por no cumplir los criterios de conducta antisocial adscritos al PCL-R, instrumento más utilizado para su evaluación en prisiones. Desde una perspectiva práctica, si bien el mantenimiento de esta alta exigencia para establecer la presencia de psicopatía (además de presentar una personalidad psicopática, deben presentar una carrera criminal) permite que los prejuicios y dificultades asociadas a este etiquetamiento se limiten a aquellos que tienen un cuadro de mayor complejidad, lo cierto es que sesga nuestra comprensión del constructo y perjudica el trabajo realizado por los profesionales de los establecimientos penitenciarios.

\section{Referencias}

Andrade, J. T. (2008). The inclusion of antisocial behavior in the construct of psychopathy: A review of the research. Aggression and Violent Behavior, 13, 328-335. doi: 10.1016/j.avb.2008.05.001

Auty, K. M., Farrington, D. P. y Coid, J. W. (2015). Intergenerational transmission of psychopathy and mediation via psychosocial risk factors. The British Journal of Psychiatry, 206(1), 26-31. doi: 10.1192/ bjp.bp. 114.151050

Babiak, P. y Hare, R. D. (2006). Snakes in suits: When psychopaths go to work. New York: Regan Books.

Cleckley, H. (1976). The mask of sanity: An attempt to clarify the so-called psychopathic personality. St. Louis, MO: C.V. Mosby.

Cooke, D. J., Hart, S. D., Logan, C. y Michie, C. (2012). Explicating the Construct of Psychopathy: Development and Validation of a Conceptual Model, the Comprehensive Assessment of Psychopathic Personality (CAPP). International Journal of Forensic Mental Health, 11, 242-252. doi: 10.1080/14999013.2012.746759

Cooke, D. J. y Michie, C. (2001). Refining the construct of psychopathy: towards a hierarchical model. Psychological Assessment, 13, 171-188. doi: 10.1037/1040-3590.13.2.171

Cooke, D. J., Michie, C., Hart, S. D. y Clark, D. A. (2004). Reconstructing psychopathy: Clarifying the significance of antisocial and socially deviant behavior in the diagnosis of psychopathic personality disorder. Journal of Personality Disorders, 18, 337-357. doi: 10.1521/ pedi.18.4.337.40347

Cooke, D. J., Michie, C. y Skeem, J. (2007). Understanding the structure of the Psychopathy Checklist-Revised: An exploration of methodological confusion. The British Journal of Psychiatry, 190, 39-50. doi: 10.1192/bjp.190.5.s39

Declercq, F., Willemsen, J., Audenaert, K. y Verhaeghe, P. (2012). Psychopathy and predatory violence in homicide, violent, and sexual offences: Factor and facet relations. Legal and Criminological Psychology, 17, 59-74. doi: 10.1348/135532510X527722

DeMatteo, D., Edens, J. F., Galloway, M., Cox, J., Smith, S. T., Koller, J. P. y Bersoff, B. (2014). Investigating the role of the psychopathy Checklist-Revised in united states case law. Psychology, Public Policy, and Law, 20(1), 96-107. doi: 10.1037/a0035452

DeMatteo, D., Heilbrun, K. y Marczyk, G. (2005). Psychopathy, risk of violence, and protective factors in a noninstitutionalized and noncriminal sample. The International Journal of Forensic Mental Health, 4, 147-157. doi: 10.1080/14999013.2005.10471220

DeMatteo, D., Heilbrun, K., y Marczyk, G. (2006). An empirical investigation of psychopathy in a noninstitutionalized and noncriminal sample. Behavioral Sciences \& the Law, 24(2), 133-146. doi: 10.1002/bsl.667

Estrada, C., Rodríguez-Díaz, F. J. y Solano, C. (2012). Castigo y carrera delictiva de liberados del sistema penitenciario de Jalisco. En S. P. Colín Soto y E. García López (Dir.), Más allá de la Violencia. Retos hacia la reconstrucción (pp. 291-320). Morelia, México: UDEM (Universidad de Morelia).

Farrington, D. P. (2006). Family background and psychopathy. En C. J. Patrick (Ed.), Handbook of Psychopathy (pp. 229-.250). New York: Guildford Press.

García, J., Arango, J. C., Correa, O., Pérez, A. F., Hugo, V., Mejía, C. A., Casals, S., López, G. J., Patiño, J. D. y Palacios, C. A. (2008). Validación de la Lista de chequeo de psicopatía-revisada (PCL-R) en población carcelaria masculina de Colombia. Revista Colombiana de Psiquiatría, 37, 564-579.

García, C. H., Moral, J., Frías, M., Valdivia, J. A. y Díaz, H. L. (2012). Family and socio-demographic risk factors for psychopathy among prison inmates. The European journal of psychology applied to legal context, 4, 119-134.

Garrido, V. G. (2002). El tratamiento del psicópata. Psicothema, 14, 181-189.

Garrido, V. G. (2004). Cara a cara con el psicópata. Barcelona: Ariel

Guy, L. S., Kusaj, C., Packer, I. K. y Douglas, K. S. (2015). Influence of the HCR-20, LS/CMI, and PCL-R on decisions about parole suitability 
among lifers. Law and Human Behavior, 39(3), 232-243. doi: 10.1037/ lhb0000111

Hall, J. R. y Benning, S. D. (2006). The "successful" psychopath: Adaptive and subclinical manifestations of psychopathy in the general population. En C. J. Patrick (Ed.), Handbook of Psychopathy. New York: Guilford Press.

Hare, R. D. (1991). Manual for the Revised Psychopathy ChecklistRevised (1st Ed.). Toronto, Ontario, Canada: Multi-Health Systems.

Hare, R. D. (1993). Without conscience: The disturbing world of the psychopaths among us. New York: Pocket Books.

Hare, R. D. (2003). Manual for the Revised Psychopathy ChecklistRevised (2nd Ed.). Toronto, Ontario, Canada: Multi-Health Systems.

Hart, S. D., Cox, D. N. y Hare, R. D. (1995). The Hare Psychopathy Checklist: Screening Version $\left(1^{a} E d\right.$.). Toronto: Multi-Health Systems.

Juarros-Basterretxea, J., Robles-Fernández, A., Silvana M., Gutiérrez, A. y García-Cueto, E. (2013). Escalamiento subjetivo de conductas delictivas. Revista Electrónica de Metodología Aplicada, 18, 37-52.

Krischer, M. K. y Sevecke, K. (2008). Early traumatization and psychopathy in female and male juvenile offenders. International Journal of Law and Psychiatry, 31, 253-262. doi: 10.1016/j.ijlp.2008.04.008

Levenson, M. R., Kiehl, K. A. y Fitzpatrick, C. M. (1995). Assessing psychopathic attributes in a non institutionalized population. Journal of Personality and Social Psychology, 68, 151-158. doi: 10.1037/00223514.68.1.151

Lilienfeld, S. O. y Andrews, B. P. (1996). Development and preliminary validation of a self-report measure of psychopathic personality traits in noncriminal populations. Journal of personality assessment, 66, 488-524. doi: 10.1207/s15327752jpa6603_3

Lloyd, C. D., Clark, H. J. y Forth, A. E. (2010). Psychopathy, expert testimony, and indeterminate sentences: Exploring the relationship between Psychopathy Checklist-Revised testimony and trial outcome in Canada. Legal and Criminological Psychology, 15(2), 323-339. doi: $10.1348 / 135532509$ X468432

López, R. (2010). Déficit en el sistema motivacional aversivo en psicópatas subclínicos evaluados mediante el Psychopathic personality inventoryrevised (PPI-R). Castellón: Universtat Jaume I. Tesis de licenciatura no publicada.

Lykken, D. (1995). The antisocial personalities. New Jersey: Lawrence Erlbaum Associates, Inc. Publishers.

Lynam, D. R., Loeber, R. y Stouthamer-Loeber, M. (2008). The stability of psychopathy from adolescence into adulthood: The search for moderators. Criminal Justice and Behavior, 35, 228-243. doi: 10.1177/0093854807310153

Molinuevo, B., Pardo, Y., González, L. y Torrubia, R. (2014). Memories of parenting practices are associated with psychopathy in juvenile male offenders. Journal of Forensic Psychiatry \& Psychology, 25(4), 495500. doi: 10.1080/14789949.2014.920901

Moltó, J., Poy, R. y Torrubia, R. (2000). Standarization of the Hare Psychopathy Checklist-Revised in a Spanish prison sample. Journal of Personality Disorders, 14, 84-96. doi: 10.1521/pedi.2000.14.1.84

McCarthy, L., Huband, N., Patel, S., Banerjee, P. y Duggan, C. (2012). Personality disorder and psychopathy as predictors of psychosocial and criminological outcome in mentally disordered offenders. The International Journal of Forensic Mental Health, 11, 227-237. doi: 10.1080/14999013.2012.739262

Olver, M. E. y Wong, S. C. P. (2015). Short- and long-term recidivism prediction of the PCL-R and the effects of age: A 24-year followup. Personality Disorders: Theory, Research, and Treatment, 6(1), 97-105. doi: 10.1037/per0000095

Patrick, C. J., Fowles, D. y Krueger, R. F. (2009). Triarchic conceptualization of psychopathy: Developmental origins of disinhibition, boldness, and meanness. Development and Psychopathology 21, 913-938. doi: 10.1017/S0954579409000492

Paulhus, D. L., Neumann, C. S. y Hare, R. D. (2006). Manual for the Hare Self-Report Psychopathy scale. Toronto, ON, Canada:MultiHealth Systems.

Pérez, B. (2014). Estudio de una muestra penitenciaria española y latinoamericana de la realidad de la psicopatía en prisión (Tesis doctoral,
Universidad de Oviedo, Asturias, España). Recuperado en: http:/ digibuo.uniovi.es/dspace/handle/10651/29236

Pérez, B., Herrero, J., Velasco, J. y Rodríguez-Díaz, F. J. (2015). A contrastive analysis of the factorial structure of the PCL-R: which model fits best the data. The European Journal of Psychology Applied to Legal Context, 7, 23-30. doi: 10.1016/j.ejpal.2014.10.001

Pinel, P. (1801). Traité médico-philosophique sur l'aliénation mentale ou la manie. Paris: J. A. Brosson.

Poythress, N. G., Skeem, J. L. y Lilienfeld, S. O. (2006). Associations among early abuse, dissociation, and psychopathy in an offender sample. Journal of Abnormal Psychology, 115, 288-297. doi: 10.1037/0021843X.115.2.288

Pozueco, J. M. (2010). Psicópatas integrados: Perfil psicológico y personalidad. Madrid: EOS.

Pozueco, J. M., Romero, G. y Casas, N. (2011). Psicopatía, violencia y criminalidad: un análisis psicológico-forense, psiquiátrico-legal y criminológico (Parte I). Cuaderno Medicina Forense, 17, 123-136.

Salekin, R. T. y Lochman, J. E. (2008). Child and adolescent psychopathy: The search for protective factors. Criminal Justice and Behavior, 35, 159-172. doi: 10.1177/0093854807311330

Salvador B., Arce, R y Rodríguez-Díaz, F. J. (2017). Evaluación psicométrica de la psicopatía: Una revisión meta-analítica. Revista Latinomericana de Psicología. Aceptado y pendiente de publicación.

Skeem, J. L. y Cooke, D. J. (2010). Is criminal behavior a central component of psychopathy? Conceptual directions for resolving the debate. Psychological Assessment, 22, 433-445. doi: 10.1037/a0008512

Sreenivasan, S., Walker, S. C., Weinberger, L. E., Kirkish, P. y Garrick, T. (2008). Four-facet PCL-R structure and cognitive functioning among high violent criminal offenders. Journal of Personality Assessment, 90 , 197-200. doi: 10.1080/00223890701845476

Spaans, M., Barendregt, M., Haan, B., Nijman, H. y de Beurs, E. (2011). Diagnosis of antisocial personality disorder and criminal responsibility. International Journal of Law and Psychiatry, 34, 374-378. doi: 10.1016/j.ijlp.2011.08.008

Torrubia, R., Poy, R., Moltó, J., Grayston, P. R. y Corral, S. (2010). PCLR. Escala de Evaluación de Psicopatía de Hare revisada. Manual. Madrid: TEA Ediciones.

Torrubia, R. y Cuquerella, A., (2008). Psicopatía: una entidad clínica controvertida pero necesaria en psiquiatría forense. Revista española de medicina legal, 34, 25-35. doi: 10.1016/S0377-4732(08)70023-3

Vinet, E. V. (2010). Psicopatía infanto-juvenil: avances en conceptualización, evaluación e intervención. Terapia psicológica, 28, 109-118. doi: 10.4067/S0718-48082010000100010.

Zúñiga, D., Vinet, E. V. y León, E. (2011). Caracterización Psicométrica del Psychopathy Checklist: Youth Version (PCL:YV) en Adolescentes Chilenos. Terapia psicológica, 29, 25-31. doi: 10.4067/S071848082011000100003 
